
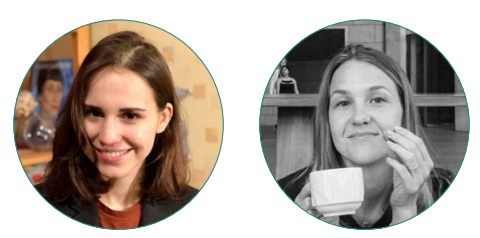

Е. Е. Булычева, Д. В. Мальцева

\title{
ВЫДЕЛЕНИЕ АКТУАЛЬНЫХ ТЕМАТИК В СОЦИОЛОГИИ: ВЗГЛЯД СКВОЗЬ ПРИЗМУ АНАЛИЗА СЕТИ ЦИТИРОВАНИЙ
}

\section{Правильная ссылка на статью:}

Булычева Е.Е., Мальцева Д.В. Выделение актуальных тематик в социологии: взгляд сквозь призму анализа сети цитирований // Мониторинг общественного мнения: экономические и социальные перемены. 2020. № 6. С. 113-140. https://doi.org/10.14515/ monitoring.2020.6.971.

\section{For citation:}

Bulycheva E. E., Maltseva D. V. (2020) Highlighting Key Topics in Sociology: A Glance Through the Prism of Citation Network Analysis. Monitoring of Public Opinion: Economic and Social Changes. No. 6. P. 113-140. https://doi.org/10.14515/monitoring.2020.6.971. (In Russ.) 
ВЫДЕЛЕНИЕ АКТУАЛЬНЫХ ТЕМАТИК В СОЦИОЛОГИИ: ВЗГЛЯД СКВОЗЬ ПРИЗМУ АНАЛИЗА СЕТИ ЦИТИРОВАНИЙ

БУЛЫЧЕВА Екатерина Евгеньевна магистр прикладной математики и информатики, Национальный исследовательский университет «Высшая школа экономики", Москва, Россия E-MAIL: e.bulycheva@protonmail.com https://orcid.org/0000-0002-2075-8527

МАЛЬЦЕВА Дарья Васильевна - кандидат социологических наук, заместитель заведующего Международной лабораторией прикладного сетевого анализа, Национальный исследовательский университет “Высшая школа экономики", Москва, Россия

E-MAIL:dmaltseva@hse.ru https://orcid.org/0000-0003-1789-1711

Аннотация. Целью работы является выделение актуальных тематик в социологии XX - начала XXI века посредством анализа публикаций в десяти ведущих англоязычных общесоциологических журналах. Исследование выполнено в количественной парадигме изучения научного знания и основано на анализе библиографической информации о публикациях из базы данных Web of Science (более 23 тыс. публикаций). Собранные данные стали основой для построения сети цитирований между публикациями. Используя метрики сетевого анализа и алгоритм выделения основного пути в этой сети, мы получаем работы с наибольшим количеством цитирований, которые можно считать наиболее значимыми для авторов отобранных журналов, прослеживаем связи между статьями и делаем выводы об актуальных тематиках исследований, преобладающих
HIGHLIGHTING KEY TOPICS IN SOCIOLOGY: A GLANCE THROUGH THE PRISM OF CITATION NETWORK ANALYSIS

Ekaterina E. BULYCHEVA ${ }^{1}$ - Master in Applied Mathematics and Computer Science

E-MAIL: e.bulycheva@protonmail.com https://orcid.org/0000-0002-2075-8527

Daria V. MALTSEVA ${ }^{1}$ - Cand. Sci. (Soc.), Deputy Head of the International Laboratory for Applied Network Research

E-MAIL:dmaltseva@hse.ru

https://orcid.org/0000-0003-1789-1711

\footnotetext{
1 National Research University Higher School of Economics, Moscow, Russia
}

Abstract. The work attempts to highlight the key topics in the sociology of $20^{\text {th }}$ early $21^{\text {st }}$ centuries by analyzing publications which appeared in ten leading general sociology journals in the English language. The study is based on a quantitative paradigm and analyzes bibliographic information on Web of Science publications (more than 23 thousand). The data collected served to build a network of citations between publications. Using the network analysis metrics and key-route main path algorithm the authors identify the publications with the highest number of citations which can be considered as the most significant for the authors of the selected journals. The article also traces the connections between the publications and highlights the topics relevant and dominant in a particlular area are relevant and dominant in a particular area of sociology in a particular time frame. 
в определенном сегменте социологии

в тот или иной период времени.

Ключевые слова: история науки, история социологии, сеть цитирований, библиометрический анализ, сетевой анализ, main path, key-routes, web of science

Благодарность. Исследование финансировалось в рамках государственной поддержки ведущих университетов Российской Федерации «5-100».

Авторы выражают благодарность анонимным рецензентам за комментарии к предыдущим версиям статьи, а также Станиславу Моисееву (НИУ ВШЭ) за идеи по улучшению текущей версии текста.
Keywords: history of science, history of sociology, citation network, bibliometric analysis, network analysis, main path, key-routes, web of science

Acknowledgments. The study was funded by the Russian Academic Excellence Project '5-100'. The authors thank the anonymous reviewers for comments on the previous versions of the article, as well as Stanislav Moiseev (HSE) for the ideas concerning the improvement of the current version.

\section{Введение}

Начиная с середины XX века социология как наука претерпевает значительные изменения. Отказ от попытки построения макротеории как единой теоретической рамки для всей социологической науки привел к стремительному увеличению числа теоретических моделей в 1960-х годах [Turner, 2001]. Теоретический плюрализм способствовал росту фрагментации, что сказалось на диверсификации не только направлений исследований, но и структуры научного сообщества. Так, количество секций в рамках Американской социологической ассоциации в период с 1960 по 1987 г. увеличилось в пять раз [Huber, 1995]. По мнению Р. Коллинза, критика доминирующих теорий, рост институционального давления на производительность ученого, изменения в методологии исследований и другие эффекты повлияли на создание множества самостоятельных специализаций в социологии, которые пользуются собственными методами исследования, теоретическим обоснованием и стандартами оценки работы [Collins, 1994]. Подобное теоретическое многообразие приводит к усложнению понимания существующих направлений и идей в социологии, их развития и преемственности. В этом контексте интересной представляется задача по изучению различных тематик исследований, присутствующих в социологической науке в целом или ее отдельных сегментах.

Изучение концептов и идей невозможно без знания их истории, необходимого для понимания причин их появления. Как утверждал П. Слоан, главной функцией истории науки является прояснение существующих в академическом знании идей с точки зрения того, что предшествовало их возникновению [Sloan, 1985]. 
Традиционный подход к изучению истории науки представляет собой качественный анализ, в ходе которого историк прослеживает в работах ученых интеллектуальные связи [Small, Garfield, 1985]. Однако если основные достижения науки легко выявляются историками, то менее значимые вклады в научный прогресс и даже относительно важные открытия могут быть упущены из виду по причине изобилия данных, подлежащих оценке. Актуальным в этом подходе всегда остается вопрос субъективности эксперта, которая, хоть и может быть нивелирована различными способами структурирования изучаемой информации, все равно проявляется в исследованиях науки качественными методами.

По мнению Д. де Солла Прайса, одного из создателей наукометрии, ученым необходима возможность изучать историю теми же методами, которые обычно применяются к физическим явлениям с целью раскрытия скрытых в них закономерностей [De Solla Price, 1970]. Такую возможность дает библиометрический анализ, основанный на количественном подходе к изучению научных публикаций с использованием математических и статистических методов и в связи с этим лишенный зависимости от субъективных взглядов ученого. Одно из направлений этого вида анализа базируется на теоретико-методологических основаниях анализа социальных сетей $\left(\mathrm{SNA}^{1}\right)$ и предполагает изучение сетей цитирований, содержащих информацию о том, какие работы были использованы для проведения исследований, для построения "цепочек научной преемственности" в различных дисциплинарных областях.

Настоящее исследование выполнено в количественной парадигме изучения научного знания и нацелено на выявление преемственности ключевых тематик в социологии XX - начала XXI в. методами библиометрического анализа. Понимая амбициозность и невозможность изучения всей социологической науки, ввиду ее многогранности и комплексности, ключевые тематики выделяются нами на основе анализа публикаций в десяти ведущих англоязычных общесоциологических журналах, отобранных по объективным критериям. Мы предполагаем, что наша работа может быть интересна не только с содержательной, но и с методологической точки зрения, поэтому подробно останавливаемся на изложении используемой методологии. В следующих частях статьи описаны теории цитирования и метод анализа сетей цитирований, используемый в нашем исследовании, а также представлены его база данных и основные результаты. Используя метрики сетевого анализа и алгоритм определения основных путей в сети цитирования, мы выделяем работы, имеющие наибольшее количество цитирований, прослеживаем связи статей друг с другом и делаем выводы об актуальных тематиках исследований, преобладающих в тот или иной период времени в определенном нами сегменте социологии. В дополнение к статье приводится приложение, опубликованное онлайн, в котором указаны публикации, отобранные с помощью используемых алгоритмов.

\section{Теории цитирования}

Использование показателей цитирования в библиометрическом анализе берет свое начало в 1964 г., когда Ю. Гарфилд представил разработанный им Индекс

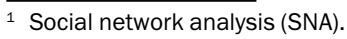


научного цитирования [Garfield, 1964]. Данный показатель впервые дал ученым и историкам науки возможность увидеть, как авторы используют другие статьи в качестве источников, на которые ссылаются в исследованиях. Как утверждал сам Ю. Гарфилд, введение этого индекса стало поворотным моментом в восприятии роли цитирования в научных работах [Garfield, Sher, Torpie, 1965].

По мнению историка науки Г. Смолла, ссылка в тексте представляет собой символ заимствованной из публикации идеи, на которую ссылаются, а сама публикация, содержащая эту идею, является лишь объектом, который ее содержит [Small, 1978]. Процесс цитирования - это обращение не ко всей работе, а лишь к идее или концепту, описанному в ней; вставляя ссылку, автор "обрамляет" ее контекстом, в котором он описывает эту идею. Данный контекст - это ее интерпретация, и для последующего читателя от первоначальной публикации доходит лишь он. Ссылаясь, ученый создает для цитируемой работы новое значение, которое теперь существует отдельно от первоначальной публикации и ее автора, но в то же время является ее символом. Г. Смолл утверждал, что цитирование служит языком, состоящим из идей и концептов, которыми оперируют ученые, создавая и описывая новое знание.

Цитирование представляет собой процесс постоянной переинтерпретации идей, которым в каждой новой ссылке придаются новые значения. Ссылаясь на определенную работу, ученый не гарантирует, что он использует заимствованные концепты в том же смысле, что и ее автор, однако таким образом он продлевает работе "жизнь".

Социальный антрополог Е. Лич, говоря о символах, присваиваемых идеям в процессе цитирования, разделил их на два типа - "разовый" и "стандартный" [Leach, 1976]. Большинство ссылок в научных публикациях представляют собой конкретную интерпретацию идеи, которая, возможно, не совпадает с интерпретацией остальных, или же вовсе ссылается на концепт, к которому ранее не обращались. Это "разовые» символы: каждый новый цитирующий автор может рассматривать одну и ту же идею в различных контекстах. "Стандартные» символы представляют собой такой вид цитирования, когда среди ученых присутствует некая конвенция по поводу интерпретации конкретной идеи. Единичное использование идеи в конкретном контексте не может сконструировать "стандартный" символ - он появляется лишь в результате объединения множественных одинаковых интерпретаций. Подобные символы легко выявить: они всегда представляют собой ссылки на высокоцитируемые работы, которые, как правило, были написаны задолго до цитирующей публикации. Как считал Г. Смолл, каждый ученый «несет с собой" так называемый сборник стандартных концептов и их символов, которые существуют в его научной сфере. Подобные работы являются «профессиональным инструментарием" ученого [Small, 1978].

При изучении феномена научного цитирования также важно понимать, что причины, по которым ученые ссылаются на публикации коллег в своих работах, могут быть разными. Так, Л. Смит приводит список из 15 возможных мотиваций, которыми руководствуются авторы, цитируя другие работы. Среди них присутствуют такие причины, как дань уважения первооткрывателям, исправление ошибок в чужих публикациях, критика, желание отдать должное коллегам, которые работают над 
схожими темами [Smith, 1981]. Таким образом, понимание цитат может отличаться в зависимости от мотивации: с одной стороны, они могут показать истинное научное влияние, с другой - авторы могут ссылаться на публикации по ряду причин личного характера. Важно понимать, что количественный анализ цитирований не дает возможности определять мотивации авторов; он исходит из представления об общей значимости связей между процитированными работами.

\section{Методология анализа сетей цитирований}

Метод библиометрического анализа берет начало во второй половине XX века. Он основан на применении математических и статистических методов к данным в реферативных журналах, публикациям конкретных авторов, научных коллективов, университетов или стран, и другим данным из книжных и журнальных изданий.

Применяемая в исследовании методология разработана В. Батагелем, A. Ферлигой и П. Дореаном [Batagelj et al., 2014] и уже использовалась для анализа сетей цитирования в различных областях: сетевого анализа в целом [Maltseva, Batagelj, 2019] и направлений кластеринга и блокмоделинга в частности [Batagelj et al., 2014], рецензирования [Batagelj et al., 2017], ИТ-аутсорсинга [Liang et al., 2016]. В отечественной литературе данная методология представлена при описании алгоритмического подхода к отбору источников для подготовки систематического обзора литературы [Моисеев, Мальцева, 2018].

Сеть цитирования представляет собой граф, где вершины-это публикации, а ребра - отношения цитирования между работами. Анализ этой сети подразумевает как подсчет базовых метрик (таких как степени входящей и исходящей центральности), так и более продвинутые методы анализа, такие как выделение основных путей (наиболее плотно связанных друг с другом цепочек публикаций) с помощью алгоритма Search path count (SPC) [Batagelj et al., 2014].

Важным условием проведения анализа с алгоритмом SPC является ацикличность сети - отсутствие в ней внутренних "циклов", то есть таких замкнутых последовательностей ребер, в которых конец каждого ребра совпадает с началом следующего. Циклы могут появиться в случаях, когда работа, цитирующая более ранние работы, получает цитирования от них (при ошибке в данных) или когда две работы, публикуемые параллельно, цитируют друг друга. При обнаружении таких участков сети используется специально разработанный алгоритм Preprint transformation, который создает "препринт-версию" для каждой публикации, в результате чего первая публикация цитирует препринт второй публикации, а вторая - препринт первой [Liu, Lu, 2012]. В сети цитирований, подготовленной для анализа, должны быть удалены "петли" (случаи, когда публикация ссылается на саму себя), а вес всех связей между узлами приравнен к единице.

К полученной сети применяется алгоритм Search Path Count, который для каждой конкретной связи вычисляет индикатор веса проходов, или traversal weights [Batagelj et al., 2014]. Этот индикатор отражает значимость ребра в сети, то есть вероятность того, что путь от искусственно созданного узла с наиболее поздним временем публикации (только цитирующего другие работы) к искусственно созданному узлу с наиболее поздним временем публикации (только цитируемому другими работами) будет проходить через данное ребро. При расчете индикатора 
значения могут быть нормализованы путем деления на сумму значений SPC для каждого ребра.

К сети с рассчитанными весами применяются алгоритмы выделения основного пути (Main path) или ключевых путей (Key-routes) [ibidem]. При применении алгоритма основного пути (Main path) для каждой публикации "верхнего уровня" (имеющей ссылки на другие работы, но не цитируемой другими) на основе последовательного выбора вершин сети по определенному правилу конструируется цепочка, ведущая к публикациям “нижнего уровня" (цитируемым другими работами, но не имеющим цитирований). Цепочка с максимальным значением показателя отбирается в качестве основного пути. Существует два варианта подсчета показателя: при реализации алгоритма локального поиска (Local search Main path) выделяется путь, где каждая следующая связь имеет наиболее высокий показатель индикатора веса проходов; при реализации алгоритма глобального поиска (Global search Main path) выделяется путь с максимальной суммой значений показателей индикатора веса проходов. При применении алгоритма поиска ключевых путей (Key-routes) в сети выделяется не один, а несколько возможных путей - через увеличение количества включенных в основной путь связей [Liu, Lu, 2012].

Данный подход позволяет выявлять цепочку наиболее значимых в контексте цитирования узлов (публикаций) в виде графа во времени. Определяя тематическую принадлежность публикаций, данный вид анализа позволяет определить основной путь развития и преемственности тематик в той или иной предметной области. В контексте сетей цитирования в основной путь входят те работы, которые не только были признаны значимыми и являются наиболее цитируемыми, но и ссылаются на значимые в предыдущем временном отрезке работы.

В настоящем исследовании использовано несколько методов анализа сетей цитирований. Базовый анализ сети цитирований подразумевал подсчет количества входящих и исходящих связей и выделение слабых компонентов сети. Помимо описания сети, результатом этой части анализа стало выделение наиболее цитируемых работ. Применение алгоритма SPC позволило нам выделить основные и ключевые пути (Main path, Key-routes) развития тематик в социологии. Анализ работ, вошедших в основные пути, стал базой выделения и изучения преемственности актуальных тематик исследований в социологии второй половины XX - начала XXI века.

Данные проанализированы в программе Pajek, предназначенной для анализа и визуализации больших сетей, разрабатываемой В. Батагелем, А. Мрваром и В. Де Ной [De Nooy, Mrvar, Batagelj, 2018].

\section{База данных исследования}

Источниками данных для библиометрического анализа могут выступать как базы данных научного цитирования (где поиск осуществляется по ключевым словам) [Batagelj, Ferligoj, Squazzoni, 2017], так и отдельные журналы [Hummon, Carley, 1993] или коллекции публикаций.

Построенная в рамках настоящего исследования база данных состоит из библиографических описаний работ, опубликованных в десяти ведущих международных научных журналах в области социологии. Выбор журналов осуществлялся по объек- 
тивным критериям с помощью ресурса Scimago Journal and Country Rank ${ }^{2}$, позволяющего выбирать журналы с наиболее высоким SJR показателем, который учитывает не только количество цитирований, но и качество их источников [Gonzales-Pereira, Guerrero-Bote, Moya-Anegon, 2010]. "Вес" ссылок присваивается таким образом, что ссылки, полученные из более "престижных" журналов, более ценны, чем из менее "престижных". Руководствуясь этим показателем, на первом этапе мы отобрали все журналы из топ-150 (Q1), содержащие в названии слова "sociology", "sociological", "Social» с целью отделить социологические журналы от журналов в близких областях (политологии, психологии и т.д.). На втором этапе, используя описания журналов, содержащие информацию об их принадлежности к отдельным областям социологии или социологической науке в целом, мы отобрали десять общесоциологических журналов (general sociological journals). Информация об этих журналах — год начала выпуска и количество выпусков в год-приведена в таблице 1.

Таблица 1. Общая информация об отобранных журналах

\begin{tabular}{|c|l|c|c|c|}
\hline \multicolumn{1}{|c|}{ Название журнала } & $\begin{array}{c}\text { Год } \\
\text { начала } \\
\text { выпуска }\end{array}$ & $\begin{array}{c}\text { Кол-во } \\
\text { выпусков } \\
\text { в году }\end{array}$ & $\begin{array}{c}\text { Кол-во публикаций в W0S } \\
\text { на момент поиска } \\
\text { (апрель 2018) }\end{array}$ \\
\hline 1. & American Sociological Review & 1936 & 6 & 24605 \\
\hline 2. & Annual Review of Sociology & 1975 & 1 & 15817 \\
\hline 3. & American Journal of Sociology & 1895 & 3 & 14265 \\
\hline 4. & Social Forces & 1925 & $2-4$ & 6227 \\
\hline 5. & European Sociological Review & 1985 & $3-6$ & 6049 \\
\hline 6. & Sociology & 1967 & $3-6$ & 5772 \\
\hline 7. & Current Sociology & 1952 & $2-7$ & 2562 \\
\hline 8. & British Journal of Sociology & 1950 & 4 & 1124 \\
\hline 9. & Sociological Quarterly & 1960 & 4 & 1148 \\
\hline 10. & Sociological Review & 1942 & 4 & 642 \\
\hline
\end{tabular}

Данные собирались в апреле 2018 г. при помощи ресурса Web of Science (WoS) регулярно обновляемой базы данных публикаций в индексированных научных журналах. Библиографические описания каждой проиндексированной в WoS cтатьи содержат информацию об авторах, названиях статей и журналов, предметных областях, ключевых словах, аннотациях и списках литературы, которые указаны в отдельных полях. Поисковый запрос по десяти журналам выдал информацию о 78211 записях (распределение количества публикаций по выбранным журналам представлено в табл. 1). Затем поиск был уточнен: мы оставили только статьи (рецензии и другие виды публикаций, не являющиеся предметом интереса данного исследования, исключили). В результате выгрузки базы данных были получены библиографические описания по 23904 публикациям в десяти журналах, где наиболее ранняя работа опубликована в 1899 г., а наиболее поздняя датируется 2018 г.

\footnotetext{
2 URL: https://www.scimagojr.com/ (дата обращения: 21.12.2020).
} 
Несмотря на то, что отобранные по объективным показателям журналы являются важными и во многом задают "повестку дня" в социологической науке, их выбор в качестве базы данных исследования накладывает на него ряд ограничений. Отбор десяти англоязычных (американских, европейских и британских) общесоциологических журналов оставляет за пределами анализа: 1) другие общесоциологические журналы, 2) журналы, посвященные более узким направлениям в социологии, 3) журналы, публикующие статьи на других языках, 4) научные монографии, информация о которых большей частью не представлена в базах данных научного цитирования. Таким образом, в фокусе нашего исследования оказывается выделение ключевых тематик не во всей социологической науке в целом, а в ее хоть и важном, но ограниченном сегменте - англоязычных статьях, опубликованных в десяти указанных журналах. Важно понимать, что выбор базы данных для анализа неизбежно влияет на его результаты, поэтому при их интерпретации нужно помнить о существующих ограничениях и не производить излишние генерализации.

Собранная база библиографических описаний работ была обработана с помощью программы Wos2Pajek ${ }^{3}$, предназначенной для трансформации данных из WoS в несколько сетей: одномодальную сеть цитирования, используемую для данного анализа, и двумодальные сети "публикация - автор", "публикация - журнал", "публикация - ключевые слова" (которые могут быть проанализированы отдельно).

Полученная сеть цитирований Сite состоит из 459891 публикации, из которых 23904 - это основные работы, отобранные в рамках поиска и имеющие полные библиографические описания (так называемые hits, хиты). Оставшиеся 435987 публикаций - это работы, указанные в списках цитирований основных статей (в отдельном поле CR), или цитируемые публикации. По этим работам мы имеем только ограниченный набор информации - имя первого автора, короткое название источника публикации (журнала), год издания, номер выпуска и начальной страницы. Например, для работы М. Грановеттера "Сила слабых связей", опубликованной в 1973 г. в American Journal of Sociology, описание будет следующим: GRANOVETTER M, 1973, AM J SOCIOL, V78, P1360.

Для каждой работы программа генерирует короткое имя, где указаны восемь первых букв фамилии автора и его инициалы, год публикации (в скобках), номер выпуска журнала и начальная страница публикации. Для нашего примера короткое имя будет таким: GRANOVET_M(1973)78:1360. Поскольку библиографические описания одной и той же работы могут различаться, создание таких имен позволяет минимизировать их дублирование в нескольких узлах сети. Такие короткие имена удобно использовать не только для обозначения работ, но и для визуализации результатов анализа. Для облегчения процесса их интерпретации программа Wos2Pajek также генерирует файл, "расшифровывающий” короткие имена статей, имеющих полное описание, и содержащий информацию о первом авторе, названии статьи и годе ее публикации. Для всех работ, входящих в сеть цитирования, формируется также отдельный файл с годом их публикации.

\footnotetext{
${ }_{3}^{3}$ Batagelj V. (2007). WoS2Pajek. Manual for version 1.4, July 2016. URL: http://vladowiki.fmf.uni-lj.si/doku.php?id=pajek: wos2pajek (дата обращения: 23.12.2020).
} 


\section{Результаты исследования}

Распределения публикаций по году. На рисунке 1 изображены графики распределения по годам публикаций с полным описанием, вышедших в десяти выбранных журналах (хиты), только цитируемых работ, информация о которых содержится в поле CR (цитируемые публикации), а также всех работ. Они показывают, что количество статей в десяти журналах с 2000 г. стремительно растет. Можно видеть, что количество цитируемых публикаций, вышедших в этот период, снижается, однако это обусловлено тем, что недавние статьи естественным образом цитируют те, что были опубликованы в предшествующий им период времени.
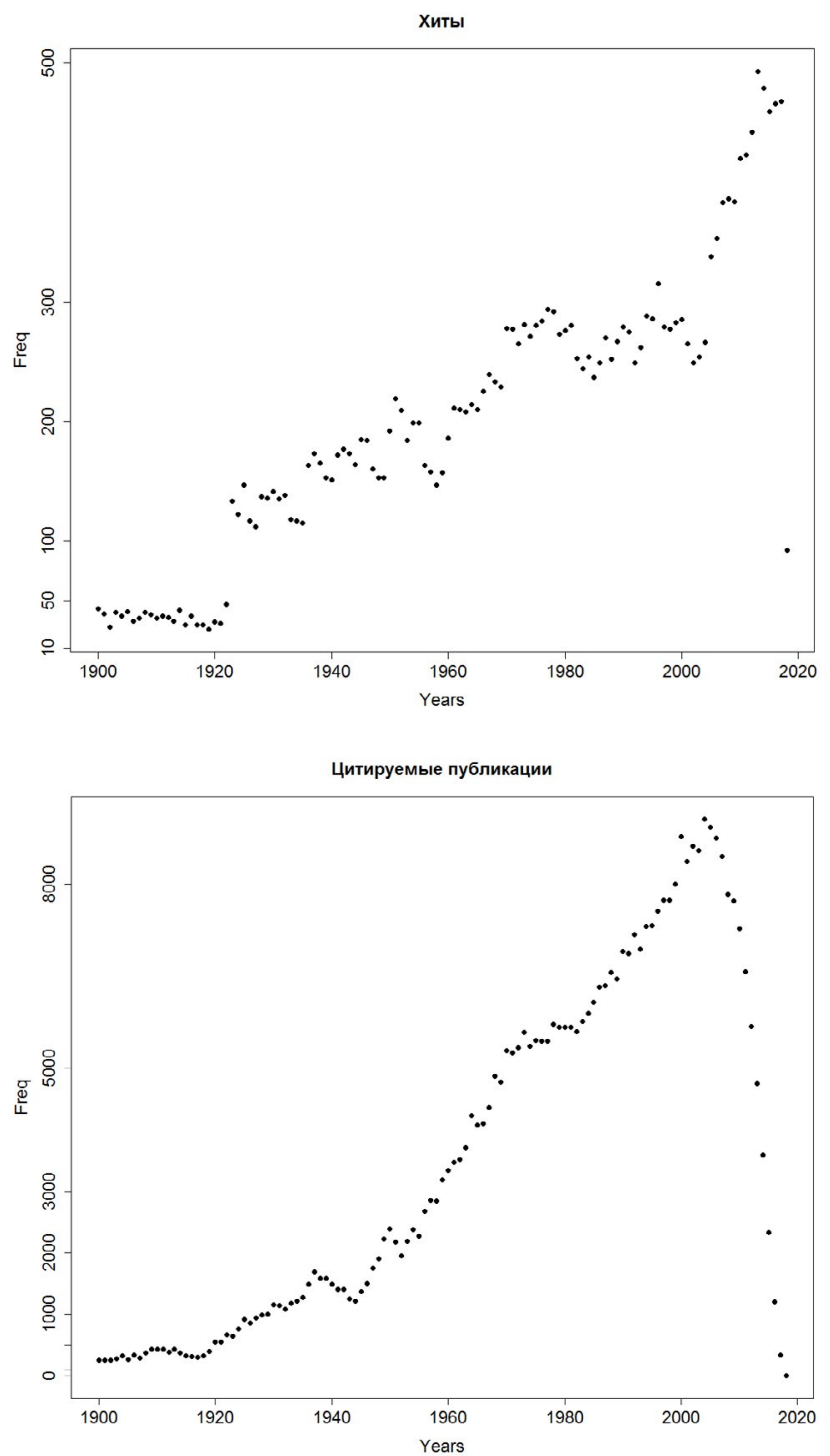


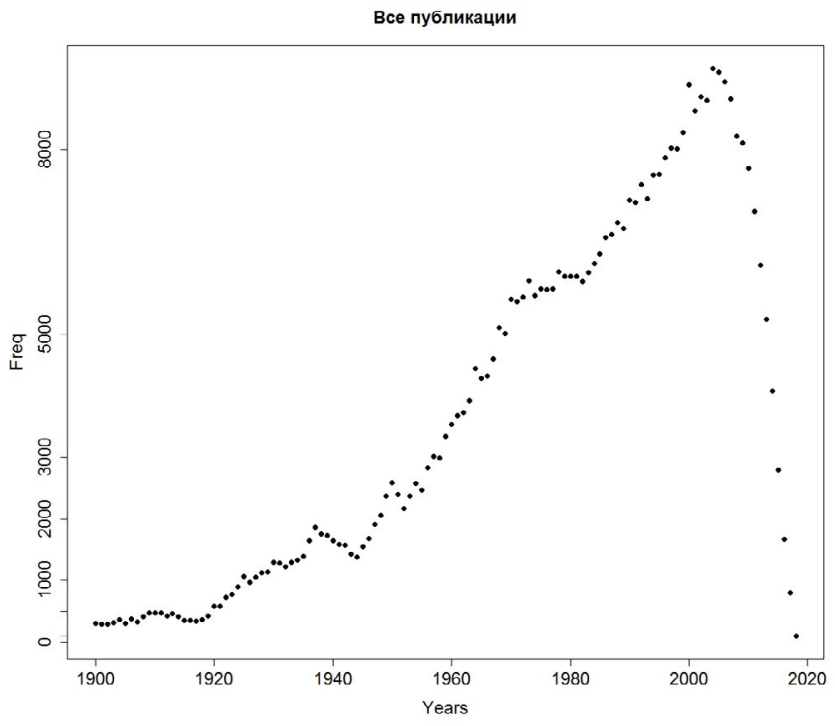

Рис. 1. Распределение публикаций по годам

Распределение количества публикаций в сети цитирований. Распределения количества входящих (indegree) и исходящих (outdegree) связей в сети цитирований (см. рис. 2) являются довольно стандартными для библиометрического анализа. Т. Низонгер [Nisonger, 2008], исследуя выполняемость закона В. Парето "20/80" на данных о библиографических ссылках, эмпирическим образом доказал, что данный принцип также работает и в научном цитировании: на $20 \%$ публикаций приходится $80 \%$ от общего числа цитирований. Подтверждение принципа В. Парето также можно увидеть и на графике показателей полученного работами цитирования (сверху): наибольшее количество цитирований приходится на небольшое количество ключевых работ.

О том же говорит и распределение цитирования, произведенного в пользу других работ (снизу) - небольшое количество статей имеет очень большое количество цитирований других публикаций, в то время как большинство статей имеют сравнительно небольшое количество цитируемых источников. Как правило, публикации, имеющие наибольшее количество произведенных в пользу других работ цитирований, - это публикации обзорного типа, диссертации и книги ${ }^{4}$. Из 40 публикаций с наибольшим количеством ссылок на другие публикации 24 принадлежат журналу Annual review of Sociology, что объясняется особенностью журнала.

\footnotetext{
4 К таким работам относятся (указаны имена авторов и названия работ на английском языке): Lebas E. (1982) Urban and Regional Sociology in Advanced Industrial Societies: a Decade of Marxist and Critical Perspectives. Current Sociology. Vol. 30. No. 1 (846 ссылок); Marx G. T., Wood J. L. (1975) Strands of Theory and Research in Collective Behaviour. Annual Review of Sociology. Vol. 1. 363-428 (379 ссылок); Williams R. M. (1975) Race and Ethnic Relations. Annual Review of Sociology. Vol. 1. 125-164 (332 ссылки); Lipman-Blumen J., Tickamyer A. R. (1975) Sex Roles in Transition: 10 Years Perspective. Annual Review of Sociology. Vol. 1. 297-337 (331 ссылка).
} 

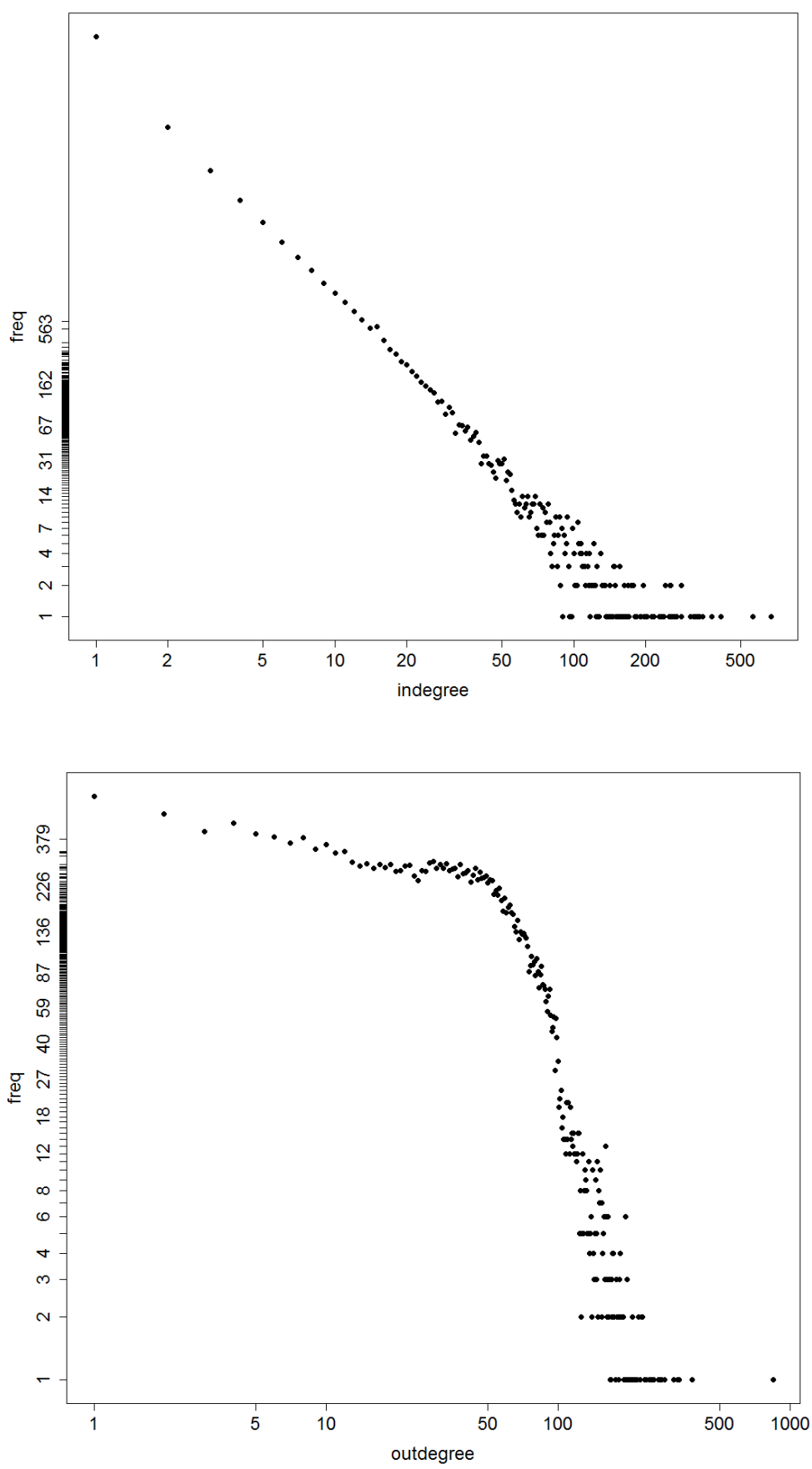

Рис. 2. Распределение числа входящих (indegree) и исходящих (outdegree) связей в сети цитирования 
Наиболее цитируемые публикации. Список из 40 наиболее цитируемых публикаций (по показателю indegree) приведен в таблице 2. Из 40 работ, наиболее часто цитируемых авторами десяти отобранных журналов, только шесть являются статьями, четыре из них опубликованы в American Journal of Sociology и две - в American Sociological Review: М. Грановеттер (M. Granovetter) ${ }^{5}$ "Сила слабых связей» (1973) ; П. Димаджио, У. В. Пауэлл (P. DiMaggio, W. Powell) «Новый взгляд на „железную клетку“: институциональный изоморфизм и коллективная рациональность в организационных полях" (1983) ${ }^{7}$; Дж. Мейер, Б. Роуэн (J. Meyer, B. Rowan) «Институционализированные организации: формальная структура как миф и церемониал" (1977) ${ }^{8}$; М. Грановеттер (M. Granovetter) «Экономическое действие и социальная структура: проблема укорененности" (1985) ${ }^{9}$; Дж. Маккарти, M. Зальд (J. McCarthy, M. Zald) «Мобилизация ресурсов и общественные движения: частичная теория" $(1977)^{10}$; Э. Свидлер (A. Swidler) “Культура в действии: символы и стратегии" (1986) ${ }^{11}$. Остальные работы - книги. Большинство наиболее цитируемых публикаций являются ранними: лишь одна из них была издана в 2000 г.

Наиболее цитируемые работы представляют собой описание различных социологических теорий (см. табл. 2): социального обмена - П. Блау (P. Blau); социального пространства и габитуса - П. Бурдье (Р. Bourdieu); структурации - Э. Гидденс (A. Giddens); структурного функционализма - Т. Парсонс (Т. Parsons) и т. Д. В таблице 2 представлены и классические работы в различных областях социологии (социальное неравенство, мобильность, социология гендера и др.), а также работы классиков социологии XIX века: М. Вебера (М. Weber) и Э. Дюркгейма (E. Durkheim), которые имеют высокие показатели входящего цитирования. Ссылки на эти публикации можно рассматривать как "стандартные символы", по Г. Смоллу, характерные для авторов десяти отобранных журналов [Small, 1978].

Таблица 2. Наиболее цитируемые социологические работы второй половины XX века

\begin{tabular}{|c|c|c|l|c|}
\hline $\begin{array}{c}\text { Кол-во } \\
\text { цитирований }\end{array}$ & Первый автор & Год & \multicolumn{1}{|c|}{ Название } & Источник \\
\hline 668 & P. Blau & 1967 & American Occupational Structure & Книга \\
\hline 563 & P. Bourdieu & 1984 & Distinction & Книга \\
\hline 412 & A. Giddens & 1991 & Modern and Self-Identity & Книга \\
\hline 378 & P. Bourdieu & 1977 & Outline of a Theory of Practice & Книга \\
\hline 346 & T. Parsons & 1951 & The Social System & Книга \\
\hline
\end{tabular}

5 Здесь и далее приводятся переводы имен авторов на русский язык с последующим указанием оригинальных имен.

6 Granovetter M. (1973) The Strength of Weak Ties. American Journal of Sociology. Vol. 78. No. 6. P. 1360-1380.

7 DiMaggio P., Powell W. (1983) The Iron Cage Revisited: Institutional Isomorphism and Collective Rationality in Organizational Fields. American Sociological Review. Vol. 48. No. 2. P. 147-160.

8 Meyer J., Rowan B. (1977) Institutionalized Organizations: Formal Structure as Myth and Ceremony. American Journal of Sociology. Vol. 83. No. 2. P. 340-363.

9 Granovetter M. (1985) Economic Action and Social Structure: The Problem of Embeddedness. American Journal of Sociology. Vol. 91. No. 3. P. 481-510.

${ }^{10}$ McCarthy J., Zald M. (1977) Resource Mobilization and Social Movements: A Partial Theory. American Journal of Sociology. Vol. 82. No. 6. P. $1212-1241$.

${ }^{11}$ Swidler A. (1986) Culture in Action: Symbols and Strategies. American Sociological Review. Vol. 51. No. 2. P. 273 -286. 


\begin{tabular}{|c|c|c|c|c|}
\hline $\begin{array}{c}\text { Кол-во } \\
\text { цитирований }\end{array}$ & Первый автор & Год & Название & Источник \\
\hline 335 & W. Wilson & 1987 & The Truly Disadvantaged & Книга \\
\hline 332 & U. Beck & 1992 & Risk Society: Towards a New Modernity & Книга \\
\hline 329 & E. Goffman & 1959 & $\begin{array}{l}\text { The Presentation of Self in Everyday } \\
\text { Life }\end{array}$ & Книга \\
\hline 323 & G. Mead & 1934 & Mind, Self and Society & Книга \\
\hline 317 & J. Coleman & 1990 & Foundations of Social Theory & Книга \\
\hline 308 & R. Putnam & 2000 & $\begin{array}{l}\text { Bowling Alone: The Collapse and } \\
\text { Revival of American Community }\end{array}$ & Книга \\
\hline 282 & $\begin{array}{l}\text { G. Espring- } \\
\text { Andersen }\end{array}$ & 1990 & The Three Worlds of Welfare Capitalism & Книга \\
\hline 281 & M. Granovetter & 1973 & The Strength of Weak Ties & $\begin{array}{c}\text { American Journal } \\
\text { of Sociology }\end{array}$ \\
\hline 281 & R. Merton & 1957 & Social Theory and Social Structure & Книга \\
\hline 270 & D. Massey & 1993 & $\begin{array}{l}\text { Theories of International Migration: } \\
\text { A Review and Appraisal }\end{array}$ & Книга \\
\hline 263 & P. J. DiMaggio & 1983 & $\begin{array}{l}\text { The Iron Cage Revisited: Institutional } \\
\text { Isomorphism and Collective Rationality } \\
\text { in Organizational Fields }\end{array}$ & $\begin{array}{l}\text { American } \\
\text { Sociological } \\
\text { Review }\end{array}$ \\
\hline 260 & H. Garfinkel & 1967 & Studies in Ethnomethodology & Книга \\
\hline 258 & P. Bourdieu & 1990 & Structures, habitus, practices & Книга \\
\hline 256 & E. Goffman & 1961 & $\begin{array}{l}\text { Asylums: Essays on the social situation } \\
\text { of mental patient and other inmates }\end{array}$ & Книга \\
\hline 254 & R. Ericson & 1992 & $\begin{array}{l}\text { The Constant Flux: A Study of Class } \\
\text { Mobility in Industrial Countries }\end{array}$ & Книга \\
\hline 254 & P. Blau & 1964 & Justice in Social Exchange & Книга \\
\hline 253 & C. Tilly & 1978 & From Mobilization to Revolution & Книга \\
\hline 255 & R. Kanter & 1977 & Men and Women of the Corporation & Книга \\
\hline 251 & A. Giddens & 1984 & The Constitution of Society & Книга \\
\hline 250 & H. Blalock & 1967 & $\begin{array}{l}\text { Toward a Theory of Minority-Group } \\
\text { Relations }\end{array}$ & Книга \\
\hline 241 & E. Goffman & 1963 & Stigma & Книга \\
\hline 241 & D. McAdam & 1982 & $\begin{array}{l}\text { The Political Process and the } \\
\text { Development of Black Insurgency }\end{array}$ & Книга \\
\hline 238 & J. Meyer & 1977 & $\begin{array}{l}\text { Institutionalized Organizations: Formal } \\
\text { Structure as Myth and Ceremony }\end{array}$ & $\begin{array}{l}\text { American Journal } \\
\text { of Sociology }\end{array}$ \\
\hline 237 & M. Weber & 1978 & $\begin{array}{l}\text { Economy and Society: An Outline of } \\
\text { Interpretive Sociology }\end{array}$ & Книга \\
\hline
\end{tabular}




\begin{tabular}{|c|c|c|l|c|}
\hline $\begin{array}{c}\text { Кол-во } \\
\text { цитирований }\end{array}$ & Первый автор & Год & \multicolumn{1}{|c|}{ Название } & Источник \\
\hline 235 & H. Braverman & 1974 & Labor and Monopoly Capital & Книга \\
\hline 234 & E. Durkheim & 1951 & Suicide: A Study in Sociology & Книга \\
\hline 230 & P. Blau & 1977 & $\begin{array}{l}\text { Inequality and Heterogeneity: } \\
\text { A Primitive Theory of Social Structure }\end{array}$ & Книга \\
\hline 228 & H. Becker & 1963 & $\begin{array}{l}\text { Outsiders: studies in the sociology of } \\
\text { deviance }\end{array}$ & Книга \\
\hline 216 & M. Granovetter & 1985 & $\begin{array}{l}\text { Economic Action and Social Structure: } \\
\text { The Problem of Embeddedness }\end{array}$ & $\begin{array}{c}\text { American Journal } \\
\text { оf Sociology }\end{array}$ \\
\hline 213 & J. McCarthy & 1977 & $\begin{array}{l}\text { Resource Mobilization and Social } \\
\text { Movements: A Partial Theory }\end{array}$ & $\begin{array}{c}\text { American Journal } \\
\text { оf Sociology }\end{array}$ \\
\hline 211 & P. Bourdieu & 1992 & Microcosms & Книга \\
\hline 202 & G. Homans & 1961 & Social Behavior: Its Elementary Forms & Книга \\
\hline 198 & J. Coleman & 1966 & Equality of Educational Opportunity & Книга \\
\hline 197 & R. Edwards & 1979 & $\begin{array}{l}\text { Contested terrain: the transformation of } \\
\text { the workplace in the twentieth century }\end{array}$ & \begin{tabular}{l} 
Книга \\
\hline 196
\end{tabular} \\
\hline A. Swidler & 1986 & $\begin{array}{l}\text { Culture in Action: Symbols and } \\
\text { Strategies }\end{array}$ & $\begin{array}{c}\text { American } \\
\text { Sociology Review }\end{array}$ \\
\hline
\end{tabular}

Редукция сети цитирований. Распределение входящей центральности (indegree) на рисунке 2 показало, что большое количество работ в сети цитирования имеет очень незначительный показатель полученного цитирования со стороны других. Эти публикации являются "случайными" и требуют удаления, так как трудно поддаются интерпретации и "утяжеляют" сеть [Batagelj et al., 2014]; для облегчения процедуры вычисления индикатора веса проходов (traversal weights) этими работами можно пренебречь. Проблема определения границы сети (boundary problem) была решена таким образом, чтобы в редуцированную сеть вошли, во-первых, все публикации из изначального поиска (23904 работ или 5,2\% всех публикаций), а во-вторых, только те статьи, количество цитирований которых выше определенного порогового значения. На основе анализа распределения было решено остановиться на пороговом значении 2 - включить в сеть только работы, имеющие три и более входящих цитирований (к которым относится 53205 работ, или 11,6\% всех публикаций). Из объединения этих кластеров была получена редуцированная сеть цитирований, содержащая 69712 узлов-публикаций (что составляет 15,2\% узлов от всей сети), имеющих 421714 связей. Подсети из двух кластеров пересекаются: 7397 работ, имеющих три и более цитирований, также имеют полное описание в WoS. Редуцированная сеть стала основой для дальнейшего анализа. Сеть была проверена на ацикличность и изменена с помощью алгоритма Preprint transformation. Количество узлов в итоговой сети составило 69739 публикаций.

Анализ структуры сети. В полученной сети была проведена проверка на наличие слабых компонентов. Под слабым компонентом понимается подграф, в котором от любой вершины можно достичь любую другую вершину, также являющуюся 
частью подграфа. Наличие в сети цитирования слабых компонентов означает, что есть группа (или несколько групп) публикаций, которые связаны цитированием внутри группы, однако не имеют связи с публикациями вне группы.

Анализ слабых компонентов в сети показал, что ее наибольший компонент включает 66196 публикаций, что составляет 94,95\% от общего количества работ. Следующий по размеру слабый компонент включает 3392 публикации (4,86\%). Остальные компоненты малочисленны и составляют 0,19\% от общего числа работ. Подобная структура компонентов означает, что в целом сеть является довольно связанной (что характерно для библиометрических сетей). В ходе дальнейшего анализа рассматривается наибольший компонент сети.

Пути трансформации тематик исследований в социологии второй половины XX века. Подсеть, полученная с помощью применения алгоритма поиска основного пути (Main path), представлена на рисунке 3. В данную подсеть входит 53 публикации, которые можно определить как наиболее значимые в контексте цитирования в рассматриваемом временном отрезке. С целью увеличения входящих в основной путь узлов и изучения возможных "ответвлений" между тематиками был также применен алгоритм поиска ключевых путей (Key-routes), результаты которого представляют расширенный путь преемственности тематик в социологии в десяти отобранных журналах (см. рис. 4). В выявленные алгоритмом ключевые пути вошло 124 работы, включая 53 работы из основного пути и 71 новую публикацию. Узлы на обоих графах обозначены посредством коротких имен, алгоритм для "расшифровки" которых приведен выше. Название, начинающееся c «=» (=Nee_V), обозначает публикацию, полученную в результате применения алгоритма Preprint transformation. Полный список названий публикаций и их авторов, которые вошли в основной и ключевые пути, расположенных в хронологическом порядке, размещен в приложении 1, опубликованном на ресурсе GitHub ${ }^{12}$.

Интересно отметить, что ни одна из работ, получивших наиболее высокое число цитирований (см. табл. 2), не вошла в список публикаций, полученный в ходе применения методов Main path и Key-routes (большинство этих работ - книги, не индексируемые в WoS; вместе с тем они могли появиться в качестве цитируемых источников "нижнего уровня"). Это еще раз подтверждает теорию Г. Смолла о "стандартных символах": работы, которые цитируются больше всего, используются в качестве определения базовых концептов, не всегда участвуя при этом в процессе становления и преобразования новых идей [Small, 1978]. В то же время некоторые авторы, упоминаемые в списке наиболее цитируемых работ (таблица 2),- Р. Мертон (R. Merton) ${ }^{13}$, М. Грановеттер (M. Granovetter) ${ }^{14}$, П. Димаджио (P. J. DiMaggio) ${ }^{15}$ - вошли в ключевые пути. Имена некоторых ученых упоминаются в полученной подсети несколько раз, чаще всего - Э. Холлингсхед (A. Hollingshead) и У. Х. Сьюэлл (W. H. Sewell), публикации которых встречаются в пути пять раз.

\footnotetext{
12 Приложение 1. Публикации, выделенные методами Main path и Key-routes. URL: https://github.com/Daria-Maltseva/ useful/blob/master/Sociology_Appendix1.pdf (дата обращения: 21. 04.2020)

${ }^{13}$ Merton R. (1938) Social Structure and Anomie. American Sociological Review. Vol. 3. No. 5. P. 672-682.

${ }^{14}$ Granovetter M. (1984) Small is Bountiful: Labor Markets and Establishment Size. American Sociological Review. Vol. 49. No. 3. P. 323-334.

${ }^{15}$ DiMaggio P.J. (2012) Network Effects and Social Inequality. Annual Review of Sociology. Vol. 38. P. 93-118.
} 


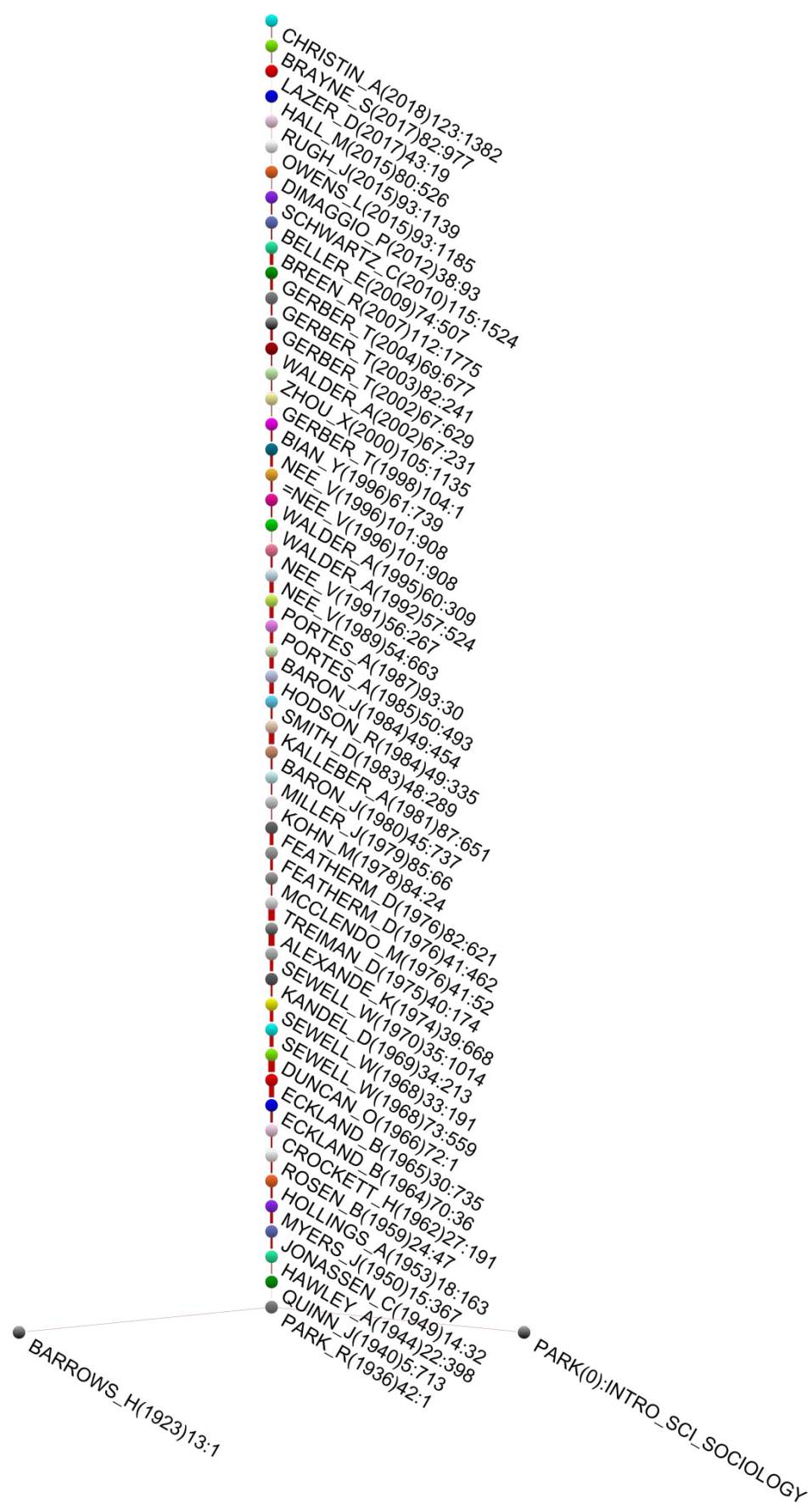

Рис. 3. Результат применения метода Main path 


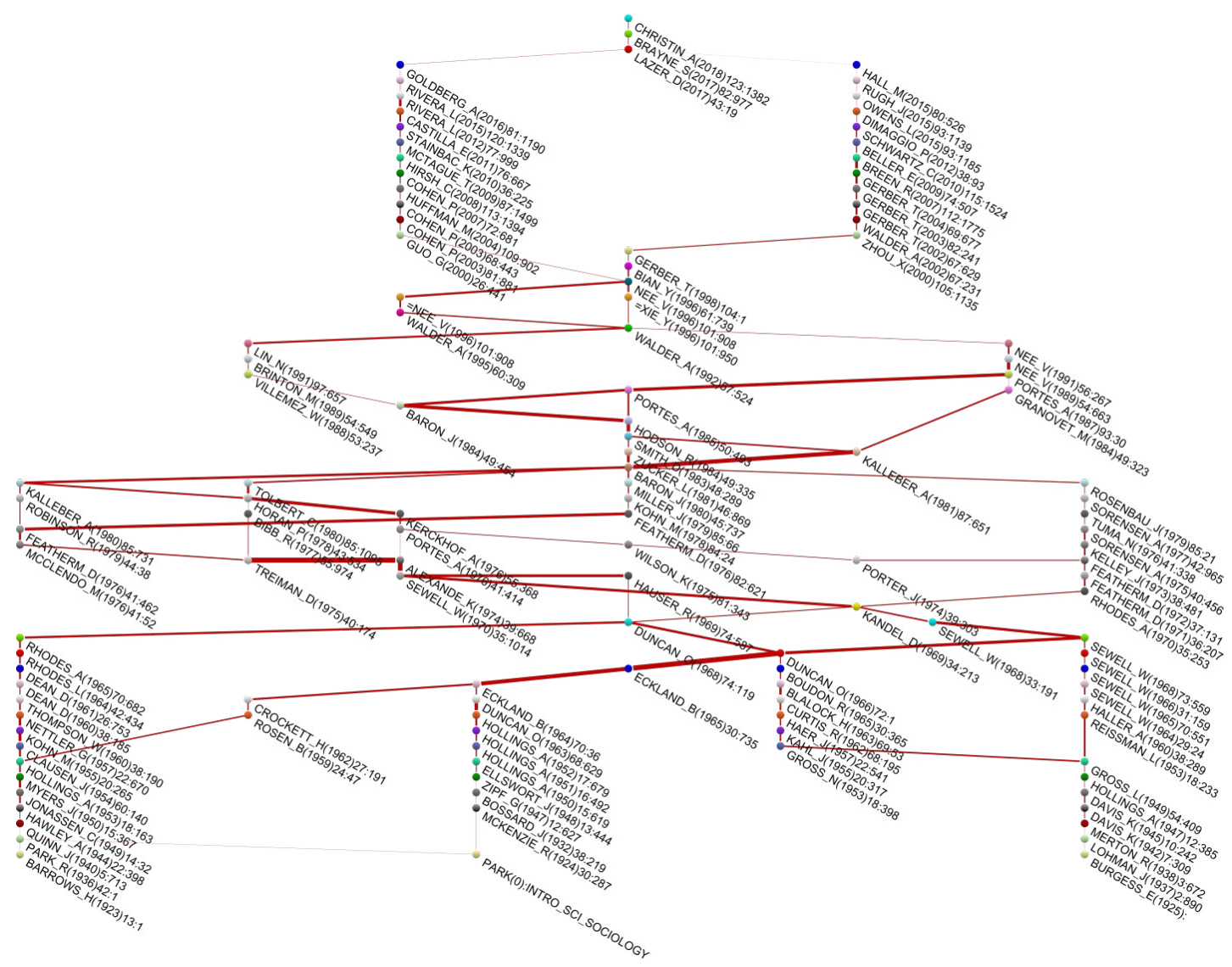

Рис. 4. Результат применения метода Key-routes

Результаты, полученные с помощью применения обоих алгоритмов, были подробно изучены. Публикации были разбиты по временным периодам, в рамках которых превалировала одна и та же тематика исследований.

Как хорошо видно на рисунке 4, публикации, относящиеся к наиболее раннему периоду, с 1920-х по 1960-е годы, формируют несколько отдельных, хоть и пересекающихся между собой, тематик исследований; они связаны с работами представителей Чикагской школы социологии. Путь слева, начинающийся с работы Г. Берроуз (H. Barrows) ${ }^{16}$, включает ряд работ по социальной экологии, проводящей параллели между социальными и экологическими системами (Р. Парк/ R. Park ${ }^{17}$, Дж. Куинн/J. Quinn ${ }^{18}$, А. Хоули/A. Hawley ${ }^{19}$ ). В более поздних работах этот подход применяется для анализа сообществ и этнических групп (К. Джонассен/

\footnotetext{
${ }^{16}$ Barrows H. (1923) Geography as Human Ecology. Annals of the Association of American Geographers. Vol. 13. No. 1. P. 1-14.

${ }^{17}$ Park R. (1936) Human Ecology. American Journal of Sociology. Vol. 42. No. 1. P. 1-15.

${ }^{18}$ Quinn J. (1940) The Burgess Zonal Hypothesis and its Critics. American Sociological Review. Vol. 5. No. 2. P. $210-218$.

${ }^{19}$ Hawley A. (1944) Ecology and Human Ecology. Social Forces. Vol. 22. No. 4. P. 398-405.
} 
C. Jonassen ${ }^{20}$ ), а также в социальной психиатрии (Дж. Клаузен/J. Clausen ${ }^{21}$ ). Затем здесь же возникают работы по темам социальной изоляции и отчуждения (М. Кон/ M. Kohn ${ }^{22}$, Г. Неттлер/G. Nettler ${ }^{23}$ ), политической апатии (Д. Дин/D. Dean ${ }^{24}$ ), аномии и их связи со статусом и сегрегацией в больших городах (А. Родес/A. Rhodes ${ }^{25}$ ).

Путь посередине, начинающийся от работы Р. Парка/R. Park и Э. Берджесс/ E. Burgess ${ }^{26}$ по введению в социологию, также связан с экологическим подходом к исследованию социальных сообществ (Р. Маккензи/R. McKenzie ${ }^{27}$ ), однако затем затрагивает вопросы брачного выбора и соседства (Дж. Боссард/J. Bossard ${ }^{28}$ ), в том числе относительно социального принципа "минимизации работы" в социальном поведении (Дж. Зипф/J. Zipf ${ }^{29}$ ). Отсюда следуют работы, посвященные социальной стратификации (А. Холлингсхед/ A. Hollingshead ${ }^{30}$ ), в том числе в связи с образовательной и профессиональной мобильностью (О.Д. Дункан/O. D. Duncan ${ }^{31}$, Б. Экланд/B. Eckland ${ }^{32}$ ). Последняя работа объединяет эту ветвь исследований с первой, через работы по мотивам (и синдрому) достижений в связи с расой и этничностью (Б. Розен/В. Rosen ${ }^{33}$, Г. Крокетт/H. Crockett ${ }^{34}$ ), которые, в свою очередь, исходят от работы по социальной стратификации (А. Холлингсхед/ A. Hollingshead ${ }^{35}$.

Путь справа начинается с работы Э. Берджесса/E. Burgess и P. Парка/R. Park ${ }^{36}$, посвященной городскому росту и экспансии, и следующим отсюда работам по социальной (дез)организации и мобильности сообществ. Последующие работы отно-

\footnotetext{
$\overline{20}$ Jonassen C. (1949) Cultural Variables in the Ecology of an Ethnic Group. American Sociological Review. Vol. 14. No. 1. $32-41$.

${ }^{21}$ Clausen J. A., Kohn M. L. (1954) The Ecological Approach in Social Psychiatry. American Journal of Sociology. Vol. 60. P. 140-151.

22 Kohn M. L., Clausen J. A. (1955) Social Isolation and Schizophrenia. American Sociological Review. Vol. 20. P. $265-273$.

${ }^{23}$ Nettler G. (1957) A Measure of Alienation. American Sociological Review. Vol. 22. No. 6. P. 670—677.

${ }^{24}$ Dean D. G. (1961) G. Alienation: its Meaning and Measurement. American Sociological Review. Vol. 26. P. $753-8$.

${ }^{25}$ Rhodes A., Reiss A., Duncan O. (1965) Occupational Segregation in a Metropolitan School System. American Journal of Sociology. Vol. 70. No. 6. P. 682-694.

${ }^{26}$ Park E., Burgess E. (1921) Introduction of the Science of Society. Chicago: University of Chicago Press.

${ }^{27}$ McKenzie R. (1924) The Ecological Approach to the Study of the Human Community. American Journal of Sociology. Vol. 30. No. 3. P. $287-301$.

${ }^{28}$ Bossard J. H. S. (1932) Residential Propinquity in Marriage Selection. American Journal of Sociology. Vol. 38. P. 219 -224

${ }^{29}$ Zipf G. (1947) The Hypothesis of the 'Minimum Equation' as a Unifying Social Principle: With Attempted Synthesis. American Sociological Review. Vol. 12. No. 6. P. 627-650.

${ }^{30}$ Hollingshead A. B. (1950) Class Differences in Family Stability. The ANNALS of the American Academy of Political and Social Science. Vol. 272. No. 1. P. 39-46.

${ }^{31}$ Duncan O. D., Hodge R. W. (1963) Education and Occupational Mobility: A Regression Analysis. American Journal of Sociology. Vol. 68. No. 6. P. 629-44.

${ }^{32}$ Eckland B. (1964) Social Class and College Graduation: Some Misconceptions Corrected. American Journal of Sociology. Vol. 70. No. 1. P. 36-50.

${ }^{33}$ Rosen B. C. (1959) Race, Ethnicity and the Achievement Syndrome. American Sociological Review. Vol. 24. P. $47-60$.

${ }^{34}$ Crockett H. (1962) The Achievement Motive and Differential Occupational Mobility in the United States. American Sociological Review. Vol. 27. No. 2. P. 191-204.

${ }^{35}$ Hollingshead A. B., Redlich F.C. (1953) Social Stratification and Psychiatric Disorders. American Sociological Review. Vol. 18. P. 163-169.

${ }^{36}$ Park R., Burgess E. (1925) The City. Chicago: The University of Chicago Press. The ANNALS of the American Academy of Political and Social Science. Vol. 124. No. 1. P. 207-207.
} 
сятся к исследованиям сообществ (Дж. Ломан/J. Lohman ${ }^{37}$ ), социальной структуре и аномии (P. Мертон/R. Merton $\left.{ }^{38}\right)$, социальной стратификации и понятию класса (Л. Гросc/L. Gross ${ }^{39}$ ). Несколько последующих работ изучают факторы влияния на образовательную и профессиональную траекторию - со стороны сверстников (А. Галлер/A. Haller ${ }^{40}$ ) и соседского сообщества (В. Сьюэлл/W. Sewell ${ }^{41}$ ). Работы, уходящие левее от уже упомянутой выше работы Л. Гросс ${ }^{38}$, продолжают развивать тематику социального класса и социально-экономических индексов (Дж. Каль/J. Kahl ${ }^{42}$, Дж. Хaep/J. Haer ${ }^{43}$ ), затем переходя к более общим темам множественных индикаторов в опросах (P. Кертис/R. Curtis ${ }^{44}$ ), латентных переменных (Г. Блэлок/H. Blalock ${ }^{45}$ ), анализа зависимостей (Р. Будон/R. Boudon ${ }^{46}$ ) и путевого анализа (О. Дункан/O. Duncan ${ }^{47}$ ). Две крайние работы каждой ветви этого направления (О. Дункан/O. Duncan ${ }^{46}$ и В. Сьюэлл/W. Sewell ${ }^{48}$ ) также оказываются связаны друг с другом.

Все три (и даже четыре, если считать расходящиеся ветви в пути справа как два) направления сходятся в нескольких работах, касающихся влияния родителей и сверстников на образовательные устремления и достижения детей (О. Дункан/ O. Duncan ${ }^{49}$, Д. Кандель/D. Kandel ${ }^{50}$ ).

В 1970-х годах направления исследований снова расходятся на разные ветви, которые в общем виде можно разделить на две части. Визуально более понятная ветвь справа продолжает тематику социальных устремлений, изучая зависимость образовательных ожиданий от религиозного контекста (А. Родес/A. Rhodes ${ }^{51}$ ),

\footnotetext{
${ }^{37}$ Lohman J. (1937) The Participant Observer in Community Studies. American Sociological Review. Vol. 2. No. 6. P. 890-897.

${ }^{38}$ Merton R. (1938) Social Structure and Anomie. American Sociological Review. Vol. 3. No. 5. P. 672-682.

${ }^{39}$ Gross L. (1949) The Use of Class Concepts in Sociological Research. American Journal of Sociology. Vol. 54. No. 5. P. 409-421.

${ }^{40}$ Haller A., Butterworth C. (1960) Peer Influences on Levels of Occupational and Educational Aspiration. Social Forces. Vol. 38. No. 4. P. $289-295$.

${ }^{41}$ Sewell W. H., Armer J. M. (1966) Neighborhood Context and College Plans. American Sociological Review. Vol. 31. No. 2. P. 159-168.

${ }^{42}$ Kahl J., Davis J. (1955) A Comparison of Indexes of Socio-Economic Status. American Sociological Review. Vol. 20. No. 3. P. $317-325$.

${ }^{43}$ Haer J. (1957) Predictive Utility of Five Indices of Social Stratification. American Sociological Review. Vol. 22. No. 5. P. $541-546$.

${ }^{44}$ Curtis R., Jackson E. (1962) Multiple Indicators in Survey Research. American Journal of Sociology. Vol. 68. No. 2. P. $195-204$.

${ }^{45}$ Blalock H. (1963) Making Causal Inferences for Unmeasured Variables from Correlations Among Indicators. American Journal of Sociology. Vol. 69. No. 1. P. 53-62.

${ }^{46}$ Boudon R. (1965) A Method of Linear Causal Analysis: Dependence Analysis. American Sociological Review. Vol. 30. No. 3. P. 365-374.

${ }^{47}$ Duncan O. (1966) Path Analysis: Sociological Examples. American Journal of Sociology. Vol. 72. No. 1. P. 1-16.

${ }^{48}$ Sewell W., Shah V. (1968) Social Class, Parental Encouragement, and Educational Aspirations. American Journal of Sociology. Vol. 73. No. 5. P. 559-572.

${ }^{49}$ Duncan O., Haller A., Portes A. (1968) Peer Influences on Aspirations: A Reinterpretation. American Journal of Sociology. Vol. 74. No. 2. P. $119-137$.

${ }^{50}$ Kandel D., Lesser G. (1969) Parental and Peer Influences on Educational Plans of Adolescents. American Sociological Review. Vol. 34. No. 2. P. 213-223.

${ }^{51}$ Rhodes A., Nam C. (1970) The Religious Context of Educational Expectations. American Sociological Review. Vol. 35. No. 2. P. $253-267$.
} 
социально-экономические достижения белых религиозно-этнических подгрупп и причинно-следственные связи между доходом и карьерой (Дж. Келли/J. Kelley ${ }^{52}$ ). Затем направление исследований переходит к изучению структуры стратификационной мобильности, возможностей карьерных достижений (например, в период роста или уменьшения компаний) и неравенства в достижениях (А. Соренсен/A. Sørensen ${ }^{53}$, Дж. Розенбаум/J. Rosenbaum ${ }^{54}$ ).

Тематика неравенства в достижениях подробно раскрывается в нескольких разрозненных ветвях направлений исследований слева. Изучается стратификационный процесс в школах (Р. Хаузер/R. Hauser ${ }^{55}$ ), гендерное (К. Александер/ K. Alexander ${ }^{56}$ ) и расовое (А. Портес/A. Portes ${ }^{57}$ ) неравенство в образовании и профессиональной среде (М. Макклэндон/M. McClendon ${ }^{58}$ ), экономическая сегментация и разница в заработках в зависимости от класса, рода занятий, сферы производства, в том числе в среде “синих воротничков" (Р. Бибб/R. Bibb ${ }^{59}$ ), а также расы (Д. Фитерман/D. Featherman ${ }^{60}$ ). Интересно отметить одно из исследований, посвященное психологическому влиянию профессиональных условий на жизнь женщин (Дж. Милер/J. Miller ${ }^{61}$ ). Большинство исследований подразумевает изучение ситуации в США, но приводится и сравнение с Великобританией (Р. Робинсон/R. Robinson ${ }^{62}$ ).

В конце 1970-х - начале 1980-х годов (работы в центре) внимание в исследованиях переходит на уровень организаций, индустрий, рынка труда и экономики в целом: институциональной дифференциации экономики, стратификации и экономической сегментации в зависимости от организации работы (Л. Цукер/L. Zucker63, Дж. Барон/J. Baron ${ }^{64}$ ), профессиональной мобильности на рынке труда (Д. Смит/

\footnotetext{
52 Kelley J. (1973) Causal Chain Models for the Socioeconomic Career. American Sociological Review. Vol. 38. No. 4. P. 481-493.

${ }^{53}$ Sørensen A. (1977) The Structure of Inequality and the Process of Attainment. American Sociological Review. Vol. 42. No. 6. P. 965-978.

${ }^{54}$ Rosenbaum J. (1979) Organizational Career Mobility: Promotion Chances in a Corporation During Periods of Growth and Contraction. American Journal of Sociology. Vol. 85. No. 1. P. 21-48.

${ }^{55}$ Hauser R. (1969) Schools and the Stratification Process. American Journal of Sociology. Vol. 74. No. 6. P. 587-611.

${ }^{56}$ Alexander K., Eckland B. (1974) Sex Differences in the Educational Attainment Process. American Sociological Review. Vol. 39. No. 5. P. $668-682$.

${ }^{57}$ Portes A. (1978) Toward a Structural Analysis of Illegal (Undocumented) Immigration. International Migration Review. Vol. 12. No. 4. P. 469-484.

${ }_{58}$ McClendon M. (1976) The Occupational Status Attainment Processes of Males and Females. American Sociological Review. Vol. 41. No. 1. P. 52-64.

${ }^{59}$ Bibb R., Form W. (1977) The Effects of Industrial, Occupational, and Sex Stratification on Wages in Blue-Collar Markets. Social Forces. Vol. 55. No. 4. P. 974-996.

${ }^{60}$ Featherman D., Hauser R. (1976) Changes in the Socioeconomic Stratification of the Races, 1962-73. American Journal of Sociology. Vol. 82. No. 3. P. 621-651.

${ }^{61}$ Miller J., Schooler C., Kohn M., Miller K. (1979) Women and Work: The Psychological Effects of Occupational Conditions. American Journal of Sociology. Vol. 85. No. 1. P. 66-94.

${ }^{62}$ Robinson R. V., Kelley J. (1979) Class as Conceived by Marx and Dahrendorf: Effects on Income Inequality and Politics in the United States and Great Britain. American Sociological Review. Vol. 44. No. 1. P. 38-58.

${ }^{63}$ Zucker L., Rosenstein C. (1981) Taxonomies of Institutional Structure: Dual Economy Reconsidered. American Sociological Review. Vol. 46. No. 6. P. 869-884.

${ }^{64}$ Baron J., Bielby W. (1980) Bringing the Firms Back in: Stratification, Segmentation, and the Organization of Work. American Sociological Review. Vol. 45. No. 5. P. 737-765.
} 
D. Smith ${ }^{65}$ ). B середине 1980-х годов появляются работы, которые обращаются к сравнению небольших и больших фирм (М. Грановеттер/M. Granovetter ${ }^{66}$, B. Уиллемез/W. Villemez ${ }^{67}$ ), неформальному сектору в западной рыночной экономике, в том числе рынку труда беженцев (А. Портес/A. Portes ${ }^{68}$ ). С конца 1980-хначала 1990-х годов направление исследований выходит за пределы западной экономики, обращаясь к тематике перехода от систем перераспределения к рыночным системам в странах социалистического устройства (И. Биан/Y. Bian ${ }^{69}$ ). На примере Китая поднимаются вопросы стратификации, социального неравенства, прав собственности, карьерной мобильности (А. Уалдер/A. Walder ${ }^{70}$ ), на примере Японии - гендерной стратификации (М. Бринтон/M. Brinton ${ }^{71}$ ). Интересно, что в 1998 г. в направлении исследований появляется статья Т. Гербера/Т. Gerber ${ }^{72}$, посвященная тем же самым вопросам - переходу к рыночной экономике, трудоустройству и доходам - но в России, в 1991-1995 гг. Затем в правой ветви исследований, опубликованных в 2000-х годах, следует еще несколько работ этого автора об экономических изменениях в нашей стране, связанных с рынком труда, образовательно-профессиональными переходами, снижением классовой мобильности. Помимо этого, в данной ветви встречается тематика изменений, происходящих в сельском и городском Китае (К. Жоу/X. Zhou ${ }^{73}$ ), а также на образовательном уровне в Швеции (P. Брин/R. Breen $\left.{ }^{74}\right)$. Исследования, идущие после, посвящены различным методологическим аспектам изучения социального неравенства влиянию положения матери (а не только отца, как было принято в исследованиях ранее) (Е. Беллер/E. Beller $\left.{ }^{75}\right)$, доходов супругов (К. Шварц/C. Schwartz $\left.{ }^{76}\right)$, эффектов социальной сети (П. Димаджио/P. DiMaggio ${ }^{77}$ ), использованию понятия класса

\footnotetext{
${ }_{65}$ Smith D. (1983) Mobility in Professional Occupational-Internal Labor Markets: Stratification, Segmentation and Vacancy Chains. American Sociological Review. Vol. 48. No. 3. P. 289-305.

${ }^{66}$ Granovetter M. (1984) Small is Bountiful: Labor Markets and Establishment Size. American Sociological Review. Vol. 49. No. 3. P. 323-334.

${ }^{67}$ Villemez W., Bridges W. (1988). When Bigger is Better: Differences in the Individual-Level Effect of Firm and Establishment Size. American Sociological Review. Vol. 53. No. 2. P. 237-255.

${ }^{68}$ Portes A., Sassen-Koob S. (1987) Making it Underground: Comparative Material on the Informal Sector in Western Market Economies. American Journal of Sociology. Vol. 93. No. 1. P. 30-61.

${ }^{69}$ Bian Y., Logan J. (1996) Market Transition and the Persistence of Power: The Changing Stratification System in Urban China. American Sociological Review. Vol. 61. No. 5. P. 739-758.

${ }^{70}$ Walder A. (1995) Career Mobility and the Communist Political Order. American Sociological Review. Vol. 60. No. 3. P. $309-328$.

${ }^{71}$ Brinton M. (1989) Gender Stratification in Contemporary Urban Japan. American Sociological Review. Vol. 54. No. 4. P. 549-564.

${ }^{72}$ Gerber T., Hout M. (1998) More Shock than Therapy: Market Transition, Employment, and Income in Russia, $1991-1995$. American Journal of Sociology. Vol. 104. No. 1. P. 1-50.

${ }^{73}$ Zhou X. (2000) Economic Transformation and Income Inequality in Urban China: Evidence from Panel Data. American Journal of Sociology. Vol. 105. No. 4. P. 1135-1174.

${ }^{74}$ Breen R., Jonsson J. (2007) Explaining Change in Social Fluidity: Educational Equalization and Educational Expansion in Twentieth-Century Sweden. American Journal of Sociology. Vol. 112. No. 6. P. 1775-1810.

${ }^{75}$ Beller E. (2009) Bringing Intergenerational Social Mobility Research into the Twenty-first Century: Why Mothers Matter. American Sociological Review. Vol. 74. No. 4. P. 507-528.

${ }^{76}$ Schwartz C. (2010) Earnings Inequality and the Changing Association between Spouses' Earnings. American Journal of Sociology. Vol. 115. No. 5. P. 1524-1557.

${ }^{77}$ DiMaggio P., Garip F. (2012) Network Effects and Social Inequality. Annual Review of Sociology. Vol. 38. P. $93-118$.
} 
(Л. Оуэнс/L. Owens ${ }^{78}$ ), а также изучению последствий экономического кризиса для роста сегрегации (Дж. Руг/J. Rugh ${ }^{79}$ ).

Исследования, опубликованные после 2000 г., расположенные слева, продолжают тематику профессиональной сегрегации и девальвации работы, выполняемой женщинами, на рынках труда США (П. Коэн/P. Cohen ${ }^{80}$ ), расового неравенства в заработной плате (М. Хаффман/M. Huffman ${ }^{81}$ ), дискриминации по полу и расе и неравенству на рабочем месте (Т. Мактак/T. McTague ${ }^{82}$ ). Несколько последующих исследований посвящены процессу принятия на работу и анализу структурного положения индивидов в организациях.

Исследование А. Голдберг и др. (A. Goldberg et al. ${ }^{83}$ ), основанное на анализе большого массива текстовых данных (писем между сотрудниками компании), и М. Холл и др. (M. Hall et al.) ${ }^{84}$, использующее инструменты симуляции данных, логичным образом соединяют две описанные ветви с несколькими работами 2017-2018 гг., посвященными использованию больших данных, их возможностям и ограничениям.

Выделенные нами тематики в обозначенном сегменте социологии в общем виде можно отобразить в виде следующей схемы (см. рис. 5). Рассматривая эти тематики, можно видеть, как изначальный интерес к изучению сообществ в рамках исследований представителей Чикагской школы социологии постепенно перетекает в изучение актуальных социальных проблем западного общества, связанных с острыми вопросами расового и гендерного неравенства в различных сферах, в том числе образовательной и профессиональной. С 1980-х годов исследования приобретают явный социально-экономический характер; фокус изучения переводится с индивидов и сообществ на уровень организаций, индустрий, рынка труда и экономики в целом (внимание к этой тематике видно и в ряде работ в списке наиболее цитируемых). В актуальной повестке дня социологов оказываются вопросы неформальных экономических отношений, "невидимые" до этого рынки и экономические отношения, а также рынки за пределами западных экономик, претерпевающие значительные трансформации. Вместе с тем тематика социального отчуждения, изоляции, сегрегации, неравенства, появившаяся в описываемом нами сегменте социологии еще в первой половине XX века, проходит через все временные периоды, приобретая новую актуальность в начале третьего тысячелетия.

\footnotetext{
${ }^{78}$ Owens L. (2015) Intrinsically Advantageous? Reexamining the Production of Class Advantage in the Case of Home Mortgage Modification. Social Forces. Vol. 93. No. 3. P. 1185-1209.

${ }^{79}$ Rugh J. (2015) Double Jeopardy: Why Latinos Were Hit Hardest by the US Foreclosure Crisis. Social Forces. Vol. 93. No. 3. P. $1139-1184$.

${ }^{80}$ Cohen P., Huffman M. (2003) Individuals, Jobs, and Labor Markets: The Devaluation of Women's Work. American Sociological Review. Vol. 68. No. 3. P. 443-463.

${ }^{81}$ Huffman M., Cohen P. (2004) Racial Wage Inequality: Job Segregation and Devaluation across U. S. Labor Markets. American Journal of Sociology. Vol. 109. No. 4. P. 902-936.

${ }^{82}$ McTague T., Stainback K., Tomaskovic-Devey D. (2009) An Organizational Approach to Understanding Sex and Race Segregation in U.S. Workplaces. Social Forces. Vol. 87. No. 3. 1499-1527.

${ }^{83}$ Goldberg A., Srivastava S., Manian V., Monroe W., Potts C. (2016) Fitting in or Standing Out? The Tradeoffs of Structural and Cultural Embeddedness. American Sociological Review. Vol. 81. No. 6. P. 1190-1222.

${ }^{84}$ Hall M., Crowder K., Spring A. (2015) Neighborhood Foreclosures, Racial/Ethnic Transitions, and Residential Segregation. American Sociological Review. Vol. 80. No. 3. P. 526-549.
} 


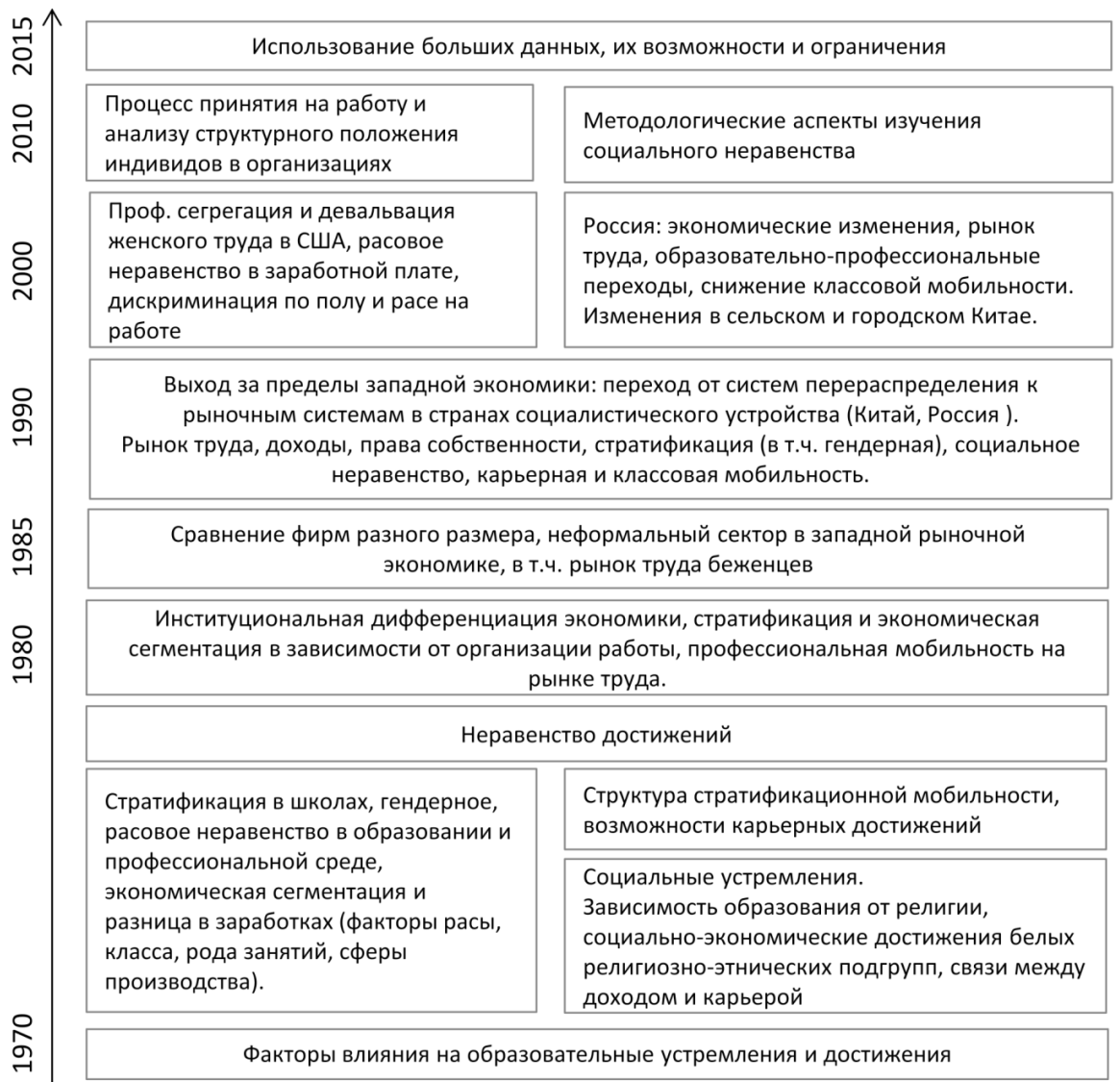

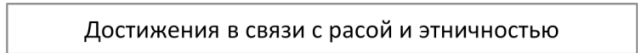

Социальная изоляция и отчуждение, политическая апатия, аномия, сегрегация

\begin{tabular}{|c|}
\hline $\begin{array}{l}\text { Брачный выбор, } \\
\text { соседство }\end{array}$ \\
Социальная экология для исследования социальных \\
сообществ
\end{tabular}
мобильность

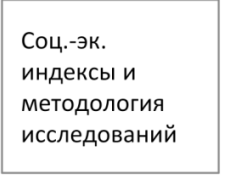

Факторы влияния на образоват. и проф.

траектории

Социальная стратификация, понятие класса

Сообщества, социальная структура, аномия

Городской рост и экспансия, соц. дезорганизация, мобильность сообществ.

Рис. 5. Схема актуальных тематик в выделенном сегменте социологического знания

На наш взгляд, ценность проведенного анализа заключается не только в том, что он позволяет выделять тематики, актуальные в тот или иной период, а также рассматривать их преемственность во времени, но и предоставляет альтернатив- 
ный взгляд на историю развития социологии. В рамках курсов по истории социологии развитие дисциплины чаще всего рассматривается как последовательность видных фигур, научных школ, состоящих из их последователей и разработанных ими теоретических подходов. В качестве альтернативы история социологии может быть понята и через последовательность тематик, привлекающих социологовисследователей и отражающих их предметную сферу интересов.

\section{Выводы}

В данной работе был проведен библиометрический анализ сети цитирования, построенной на основе публикаций в десяти ведущих англоязычных социологических журналах с целью выявления актуальных тематик исследований и их преемственности в определенном сегменте социологии XX — начала XXI века.

В результате исследования был выделен список наиболее цитируемых работ авторов, относящихся к классикам современной социологии: П. Блау, П. Бурдье, Э. Гидденс, Т. Парсонс, У. Бек, И. Гофман, Г. Мид, Дж. Коулман, Р. Патнэм, М. Грановеттер и др., а также к классикам XIX века: М. Вебер и Э. Дюркгейм. Интересно, что наиболее цитируемые публикации в социологии середины XX-начала XXI века не входят в список работ, составляющих цепочку развития тематических исследовательских направлений в данной предметной области. Согласно теории Г. Смолла, можно предположить, что эти публикации являются "стандартными символами", на которые авторы ссылаются при использовании классических теорий и концептов.

В данном исследовании нам удалось описать актуальные тематики в десяти англоязычных общесоциологических журналах, преобладающих в тот или иной период времени. Анализ публикаций, полученных с помощью алгоритмов поиска основного и ключевых путей, показал, что выделенные нами направления исследований базируются на работах представителей Чикагской школы социологии 1920-1930-х годов. Однако постепенно от социальной экологии для изучения городских сообществ и соседств тематика переходит к вопросам социальной изоляции, отчуждения, сегрегации, стратификации, аномии, влияния окружения на образовательные и профессиональные траектории (1950-1960-е годы). Тематика социальных достижений и неравенства в доступе к образованию, карьере и заработку на основе классовых, расовых и гендерных различий активно представлена и в работах 1970-х годов. В 1980-1990-е годы тематика исследований развивается в рамках экономической социологии: изучается не только рынок труда и неравенство, но и организации, индустрии, трансформирующиеся экономические системы (в том числе ситуация в России). Исследования после 2000 г. продолжают тематику неравенства, с упором на девальвацию женского труда, в том числе в связи с эффектами персональных социальных сетей индивидов. Наконец, недавние исследования 2017-2018 гг. связывают данные тематики с использованием больших данных. На наш взгляд, такой подход предоставляет альтернативный взгляд на представление истории развития социологии, поскольку рассказывает ее через последовательность тематик, привлекающих социологов-исследователей и отражающих их предметную сферу интересов. Его актуальность становится очевидной на современном этапе становления научного знания, в рамках которого развитие науки в первую очередь осуществляется и измеряется через публикации в журналах. 
Как и другие проекты, ориентированные на изучение научных дисциплин с помощью количественных методов, настоящее исследование имеет ряд ограничений, относящихся как к его базе данных, так и к проведенному анализу. В разделе с описанием базы данных уже отмечалось, что основой анализа выступили публикации из десяти англоязычных (американских, европейских и британских) общесоциологических журналов, что оставляет за пределами анализа большую часть публикаций в других источниках и других типов. Вместе с тем отобранные по объективным показателям журналы во многом задают “повестку дня" в социологической науке в целом.

Суть используемого алгоритма поиска основного и ключевых путей заключается в выделении цепочек работ, последовательно и сильно связанных друг с другом, даже если веса связей между некоторыми узлами являются не очень значительными. Поэтому другие виды библиометрического анализа, основанные на сетях соавторства, цитирования и совместного цитирования между авторами, университетами, журналами, и другими субъектами могут предоставить более детализированную картину преемственности актуальных тематик, преобладающих в определенных сегментах социологии в тот или иной период времени.

Помимо этого, мы хотим подчеркнуть важность использования качественных подходов к анализу научных дисциплин, богатая традиция которых разработана в исследованиях в области истории, философии и социологии науки. Объединение обоих подходов способно нивелировать их слабые стороны и предоставить комплексную картину развития научной дисциплины.

Результаты, полученные нами с помощью количественного анализа, могут быть интересны представителям профессионального сообщества для обзора преемственности актуальных тематик и ключевых работ определенного сегмента социологической дисциплины. Используемая в работе методология анализа может быть применена исследователями для изучения различных областей социологии и других научных направлений, а также для подготовки систематических литературных обзоров.

\section{Список литературы (References)}

Моисеев С.П., Мальцева Д. В. Отбор источников для систематического обзора литературы: сравнение экспертного и алгоритмического подходов / Социология: методология, методы, математическое моделирование (4М). 2018. № 47. С. 7-43. URL: https://www.jour.fnisc.ru/index.php/soc4m/article/view/6497/6396 (дата обращения: 21.12.2020).

Moiseev S. P., Maltseva D. V. (2018) Selection of Sources for a Systematic Literature Review: A Comparison of Expert and Algorithmic Approaches. Sociology: Methodology, Methods, Mathematical Modeling (4M). No. 47. P. 7-43. URL: https:/ /www.jour.fnisc. ru/index.php/soc4m/article/view/6497/6396 (accessed: 21.12.2020). (In Russ.)

Batagelj V., Doreian P., Ferligoj A., Kejžar N. (2014) Understanding Large Temporal Networks and Spatial Networks: Exploration, Pattern Searching, Visualization and Network Evolution. Chichester: Wiley. https://doi.org/10.1002/9781118915370. 
Batagelj V., Ferligoj A., Squazzoni F. (2017) The Emergence of a Field: A Network Analysis of Research on Peer Review. Scientometrics. Vol. 113. No. 1. P. 503-532. https://doi.org/10.1007/s11192-017-2522-8.

Collins R. (1994) Why the Social Sciences Won't Become High-Consensus, RapidDiscovery Science. Sociological Forum. Vol. 9. No. 2. P. 155-177. https://doi.org/ $10.1007 / \mathrm{bf0} 1476360$.

De Nooy W., Mrvar A., Batagelj V. (2018) Exploratory Social Network Analysis with Pajek: Revised and Expanded Edition for Updated Software. Cambridge: Cambridge University Press.

De Solla Price D. J. (1970) Citation Measures of Hard Science, Soft Science, Technology, and Nonscience. In: Nelson C. E., Pollock D. K. Communication Among Scientists and Engineers. Lexington, MA: D. C. Heath and Company. P. 3-22.

Garfield E. (1964) “Science Citation Index" - A New Dimension in Indexing. Science. Vol. 144. No. 3619. P. 649-654. https://doi.org/10.1126/science.144.3619.649.

Garfield E., Sher I. H., Torpie R. J. (1965) The Use of Citation Data in Writing the History of Science. Philadelphia, PA: Institute for Scientific Information Inc.

González-Pereira B., Guerrero-Bote V., Moya-Anegón F. (2010) A New Approach to the Metric of Journals Scientific Prestige: the SJR Indicator. Journal of Informetrics. Vol. 4. No. 3. P. 379-391. https://doi.org/10.1016/j.joi.2010.03.002.

Huber J. (1995) Institutional Perspectives on Sociology. American Journal of Sociology. Vol. 101. No. 1. P. 194-216. https://doi.org/10.1086/230702.

Hummon N. P., Carley K. (1993) Social Networks as Normal Science. Social Networks. Vol. 15. No. 1. P. 71-106. https://doi.org/10.1016/0378-8733(93)90022-d.

Leach E. (1976) Culture and Communication: The Logic by Which Symbols Are Connected. London: Cambridge University Press. https://doi.org/10.1017/cbo9780511607684.

Liang H., Wang J.-J., Xue Y., Cui X. (2016) IT Outsourcing Research From 1992 to 2013: A Literature Review Based on Main Path Analysis. Information \& Management. Vol. 53. No. 2. P. 227-251. https://doi.org/10.1016/j.im.2015.10.001.

Liu J. S., Lu Y. Y. L. (2012) An Integrated Approach for Main Path Analysis: Development of the Hirsch Index as an Example. Journal of the American Society for Information Science and Technology. Vol. 63. No. 2. P. 528-542. https://doi.org/10.1002/ asi.21692.

Maltseva D., Batagelj V. (2019) Social Network Analysis as a Field of Invasions: Bibliographic Approach to Study SNA Development. Scientometrics. Vol. 121. No. 2. P. 1085-1128. https://doi.org/10.1007/s11192-019-03193-x.

Nisonger T. E. (2008) The "80/20 Rule" and Core Journals. The Serials Librarian: From the Printed Page to the Digital Agee. Vol. 55. No. 1-2. P. 62-84. https:// doi.org/10.1080/03615260801970774. 
Sloan P. R. (1985) Essay Review: Ernst Mayr on the History of Biology. Journal of the History of Biology. Vol. 18. No. 1. P. 145-153. https://doi.org/10.1007/bf00127960.

Small H. G. (1978) Cited Documents as Concept Symbols. Social Studies of Science. Vol. 8. No. 3. P. $327-340$. https://doi.org/10.1177/030631277800800305.

Small H., Garfield E. (1985) The Geography of Science: Disciplinary and National Mappings. Journal of Information Science. Vol. 11. No. 4. P. 147-159. https://doi.org/ 10.1177/016555158501100402.

Smith L. C. (1981) Citation Analysis. Library Trend. Vol. 30. No. 1. P. 83-106. URL: http://hdl.handle.net/2142/7190 (accessed: 22.12.2020).

Turner J. H. (2001) Sociological Theory Today. In: Kaplan H. B., Turner J. H. Handbook of Sociological Theory. Handbooks of Sociology and Social Research. New York, NY: Springer. P. 1-17. https://doi.org/10.1007/0-387-36274-6_1. 